\title{
Invited Response on: Letter-to-the-Editor: The Global Prevalence of Seroma After Abdominoplasty: A Systematic Review and Meta-Analysis
}

\author{
Nader Salari ${ }^{1}$ - Behnaz Fatahi' ${ }^{2}$ Yalda Bartina ${ }^{3}$ - Mohsen Kazeminia ${ }^{2}$. \\ Mohammadbagher Heydari $^{4} \cdot$ Masoud Mohammadi $^{5}$ (D) Mahvan Hemmati $^{2}$. \\ Shamarina Shohaimi ${ }^{6}$
}

Received: 15 August 2021/Accepted: 17 August 2021/Published online: 27 August 2021

(C) Springer Science+Business Media, LLC, part of Springer Nature and International Society of Aesthetic Plastic Surgery 2021

Level of Evidence $V$ This journal requires that authors assign a level of evidence to each article. For a full description of these Evidence-Based Medicine Ratings, please refer to Table of Contents or online Instructions to Authors www.springer.com/00266.

We are very pleased that this article [1] has received such good feedback from readers, and I also thank the author of this article and the letter to the editor for their significant advice, but I need to explain the situation.

Of course, it is very important to provide a systematic review and meta-analysis to search for all sources and increase the sensitivity of the article, but sometimes in one issue, increasing the number of databases does not indicate an increase in sensitivity, but it is important to use resources that include more articles. Therefore, the authors are focused on the resources available to all authors in the

Masoud Mohammadi

Masoud.mohammadi1989@yahoo.com

1 Department of Biostatistics, School of Health, Kermanshah University of Medical Sciences, Kermanshah, Iran

2 Student research committee, Kermanshah University of Medical Sciences, Kermanshah, Iran

3 Department of Translation Studies, Faculty of Literature, Istanbul University, Istanbul, Turkey

4 Department of General Surgery, Faculty of Medicine, Kermanshah University of Medical Sciences, Kermanshah, Iran

5 Department of Nursing, School of Nursing and Midwifery, Kermanshah University of Medical Sciences, Kermanshah, Iran

6 Department of Biology, Faculty of Science, University Putra Malaysia, Serdang, Selangor, Malaysia world, and in this case, Cochrane Library is not available to everyone, and most authors are not familiar with it.

The issue of publication bias is also a very important issue that should be considered in systematic review and metanalysis studies, in which case a general picture is provided to readers using a funnel plot that cannot be interpreted by eye. Therefore, it is expected that at the same time as presenting the funnel plot, the results of the statistical test will be reported to describe the publication bias. Because in the test the results are less than 0.05 meaningful and in this case 0.06 will not be significant. In this case, the most common test that is being studied by researchers is the Egger's test, and therefore, the authors have used this test to check the publication bias.

Declarations

Conflict of interest The authors declare that they have no conflict of interest.

Human and animal rights This article does not contain any studies with human participants or animals performed by any of the authors.

Informed consent For this type of study informed consent is not required.

\section{Reference}

1. Salari N et al (2021) The global prevalence of seroma after abdominoplasty: A systematic review and meta-analysis. Aesthetic Plast Surg, https://doi.org/10.1007/s00266-021-02423-z

Publisher's Note Springer Nature remains neutral with regard to jurisdictional claims in published maps and institutional affiliations. 